\title{
Characterization of base roughness for granular chute flows
}

\author{
L. Jing and C. Y. Kwok ${ }^{*}$ \\ Department of Civil Engineering, The University of Hong Kong, Haking Wong Building, Pokfulam Road, Hong Kong \\ Y. F. Leung \\ Department of Civil \& Environmental Engineering, The Hong Kong Polytechnic University, Hong Kong \\ Y. D. Sobral \\ Departamento de Matemática, Universidade de Brasília, Campus Universitário Darcy Ribeiro, 70910-900 Brasília, DF, Brazil
}

(Received 8 June 2016; revised manuscript received 11 August 2016; published 1 November 2016)

\begin{abstract}
Base roughness plays an important role in the dynamics of granular flows but is still poorly understood due to the difficulty of its quantification. For a bumpy base made of spheres, at least two factors should be considered in order to characterize its geometric roughness, namely, the size ratio of flow to base particles and the packing arrangement of base particles. In this paper, we propose an alternative definition of base roughness, $R_{a}$, as a function of both the size ratio and the distribution of base particles. This definition is generalized for random and regular packings of multilayered spheres. The range of possible values of $R_{a}$ is presented, and optimal arrangements for maximizing base roughness are studied. Our definition is applied to granular chute flows in both two- and three-dimensional configurations, and is shown to successfully predict whether slip occurs at the base. A transition is observed from slip to nonslip conditions as $R_{a}$ increases. Critical values of $R_{a}$ are identified for the construction of a nonslip base at various angles of inclination.
\end{abstract}

DOI: 10.1103/PhysRevE.94.052901

\section{BACKGROUND}

The dense flow of granular materials has been studied in various configurations, including plane shear, annular shear, chute flows, heap flows, and surface avalanches in rotating drums [1-3]. These configurations result in either confined flows with one or more moving boundaries, or free surface flows supported by at least one substrate or base. Extensive experimental and numerical studies have illustrated the profound impact of the boundary conditions on the overall flow behavior in both two-dimensional (2D) and three-dimensional (3D) situations [2,4-25]. For instance, Pouliquen [12] found that base roughness is the key to the minimum thickness necessary to sustain a steady flow at a given inclination, and proposed a scaling law correlating this thickness with the mean flow velocity.

Unless otherwise required (e.g., frictionless side walls in $[2,26,27]$ ), nonslip condition is presumed in many studies $[1,3,12,16,28-41]$. This imposes zero velocity at the stationary base in chute flows and prohibits sliding at the moving walls in plane or annular shear flows. There are several arguments supporting the imposition of nonslip conditions. In industries, a sufficiently rough base (e.g., a conveyor belt) can maximize the transport of materials and facilitate the control of flow type [14]. By contrast, the failure of persisting nonslip condition may give rise to poorly developed shear flows, such as plug flows on frictional but planar bases [14], and inefficient energy transfer from the rotating apparatus to the flowing granular materials [24]. From the theoretical point of view, nonslip is the simplest scenario in solving the boundary value problem of granular flow modeling. It is also the most common case in geophysical situations, and is therefore widely

\footnotetext{
*fiona.kwok@hku.hk
}

adopted in the mathematical models of landslides [38,42], debris flows [43], and the segregation in shallow granular avalanches [33,44].

To achieve the nonslip base condition in physical experiments, a rough substratum is usually constructed by gluing a layer of randomly packed particles [1,12,24,31,34]. The validity of nonslip conditions may be influenced by the packing density of such particles and the deviations of their size that may occur during the manufacturing process. In numerical simulations, a layer of equal-sized spheres can be fixed beneath the flow to serve as the bumpy base $[2,14,28,35,40,45,46]$. Several arrangements of base particles have been studied [14] and it is found that neither quasiordered nor perfect-ordered bases can ensure nonslip condition. In contrast, a sufficiently rough base can be obtained with random packing that is associated with irregular bumps and interparticle spaces. In some studies of monodisperse flows, the size and distribution of base particles are set to be identical with a random layer of the flowing particles $[28,45]$. Alternatively, one can increase base roughness by fixing larger particles on the substrate [15,19-22,24].

Despite these empirical instructions on the construction of a rough base, the quantitative representation of base roughness remains rarely discussed [4-10,15]. Inspiring works on the topic include [7], where a mathematical model is developed for the $2 \mathrm{D}$ motion of a single bead on a rough inclined line, and [15], which theoretically investigates the angle of stability of a single particle on a rough plane. In both studies, different base constructions (i.e., spacing or packing) and size ratios between base particles and flow particles are considered. These indicate the possibility of defining base roughness as a function of size ratio and spacing. Such a definition would be particularly useful to better understand boundary effects in granular flows (e.g., [7,8,12,14,15,21,24]) and size segregation in bidisperse chute flows [40,46,47]. 
In the latter case, size segregation will lead to variations of relative base roughness, which affects the flow kinetics [40,46]. Meanwhile, crystallization may occur upon basal sliding, and the development of segregation may be affected [40].

In this work, basal effects in chute flows are further illustrated in Sec. II, following a brief description of the studied scenarios and the adopted numerical scheme for the current work. To characterize base roughness in a quantitative manner, an alternatively defined indicator is presented in Sec. III, including its definition, generalization to multilayer situations, and range of possible values. Phase diagrams have been constructed using the new definition for both $2 \mathrm{D}$ and $3 \mathrm{D}$ configurations. The phase diagrams can predict the slip and nonslip basal condition at a wide range of inclinations, according to the size ratio and packing of particles. Section IV discusses a few practical key points regarding the application of our indicator, including a simplified definition of base roughness that considers the packing density of base layers, and the effect of packing orientation on base roughness.

\section{BASAL CONDITION IN CHUTE FLOWS}

\section{A. Case setup}

Chute flow is an experimental paradigm of natural landslides and avalanches (Fig. 1). As a flow of granular material is continuously fed through a gate with controlled opening, a steady uniform flow of the desired thickness, $H$, is developed at a given inclination, $\theta$. The steady, fully developed (SFD) state is reached when the flow thickness, mass flow rate, and thus kinetic energy are not varying in time in the flow direction [2]. In the SFD state, the velocity profile typically obeys Bagnold's scaling and shear stress is proportional to the square of shear rate $[12,28]$. The side-wall effect is negligible if the chute is sufficiently wide, in which case the experimental setup can be simplified to a periodic sample, as adopted in many numerical simulations $[28,35]$.

The presented numerical simulations are performed using the discrete element method (DEM). As shown in Fig. 1, periodic boundaries are imposed in the flow $(x)$ and vorticity (z) directions, while a rough base is formed normal to the $y$ direction. The sample is free of constraint at the top. Spherical particles are randomly poured into the sample box under gravity, and contact properties are tuned to achieve a close packing (packing density $\sim 0.6$ ). After the sample is generated, gravity is tilted to a designed inclination, $\theta$. The inclination

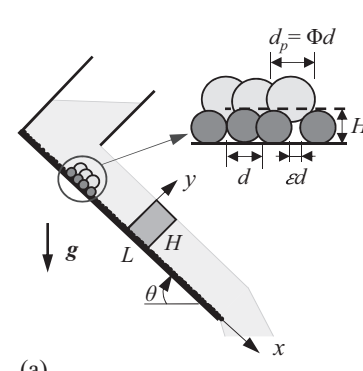

(a)

FIG. 1. Case setup. (a) Chute flow in experiments; (b) 3D and (c) 2D periodic elements in DEM. is set close to the upper limit of SFD flows [2]; i.e., $\theta=30^{\circ}$. In addition, several different inclinations are also presented in later sections for a more universal characterization of slip and nonslip conditions.

The flow particles have diameter $d_{p}=0.005 \mathrm{~m}$, with sample length $L=30 d_{p}$, width $W=10 d_{p}$, and height $H=40 d_{p}$. The sample dimensions are chosen to strike a balance between eliminating boundary effects and maximizing computational efficiency. Mechanical properties of the particles include $\rho=2500 \mathrm{~kg} / \mathrm{m}^{3}$, Young's modulus $E=5 \mathrm{MPa}$, and Poisson's ratio $v=0.35$. The contact force is calculated using the Hertz model [24,28], with normal damping given by $\gamma_{n}=\ln (e) / \Delta t$, where $e$ is the coefficient of restitution and $\Delta t$ the collision time. No tangential damping is considered. The tangential force is calculated following Coulomb friction criterion, with $\left|F_{t}\right| \leqslant\left|\mu F_{n}\right|$, where $\mu$ is the coefficient of friction, and $F_{n}$ and $F_{t}$ are normal and tangential contact forces, respectively. In the Hertz model, the tangential force is regularized at zero sliding velocity, i.e., $F_{t}=k_{t} \delta_{t}$, in which $\delta_{t}$ is tangential overlap; the tangential stiffness $k_{t}$ is a nonlinear function of normal overlap $\left(\delta_{n}\right)$ and material properties including $E$ and $v$ [28]. In the present study, the typical value of $\Delta t$ is $10^{-5} \mathrm{~s}$, which ensures numerical stability. Since the focus of the current study is placed on the geometric roughness instead of mechanical properties, $e=0.5$ and $\mu=0.5$ are consistently adopted in subsequent analyses.

\section{B. Base generation}

A variety of particle sizes and generation strategies are adopted (Table I). Main controlling variables are defined as follows: The size ratio between flow particles $\left(d_{p}\right)$ and base particles $(d)$ is denoted by $\Phi=d_{p} / d$, while the (mean) spacing measured over a layer is noted as $\varepsilon d$ [5]. Notations can be found in the magnified schematics in Fig. 1(a). Some equivalent expressions of $\varepsilon$ are the packing density of a layer, $\eta$, and compactness, $c$, which is the ratio of the area occupied by the projection of spheres within a layer to the surface area of the layer [15]. It can be derived that for a layer with all centroids coplanar, $\eta=(2 / 3) c$. A base can comprise multiple layers, in which case the packing density of its surface layer is denoted by $\eta_{s}$. The total thickness of a base is $H_{b}$, which measures the height of the bounding box of all base particles.

In Sets 1-6, different bases are generated by specifying $H_{b} / d=1.0-2.0$. If $y=0$ is defined as the top surface of a base, the generation procedure begins by firstly placing a wall at $y=-H_{b}$, then pouring base particles onto the (bottom) wall, and finally trimming particles beyond $y=0$. By varying $H_{b} / d$, surfaces with different distributions of bumps are produced on top of a dense layer (Fig. 2). In Set 7, a different strategy is used to generate the base for $\Phi=1.0$, in which a layer of particles with different packing densities, $\eta=0.2-0.6$, is randomly generated. By either approach (Sets 1-6 or Set 7), a random interparticle spacing is achieved, which provides different roughness in subsequent analyses. Figure 2 shows some typical base constructions for $\Phi=1.0$.

Other types of bases, i.e., flat plane (Set 9), and generation approaches, i.e., ordered packing (Set 8), are also presented for reference. Only monolayer, ordered bases are considered for 2D simulations (Set 11). 
TABLE I. Major simulations performed.

\begin{tabular}{lccclc}
\hline \hline Set & 2D $/ 3 \mathrm{D}$ & $\Phi$ & $\theta(\mathrm{deg})$ & & \multicolumn{1}{c}{ Base generation } \\
\hline 1 & 3D & 0.4 & 30 & & Sketch \\
2 & 3D & 0.5 & 30 & & - \\
3 & 3D & 0.67 & 30 & Random: $H_{b} / d=1.0,1.2,1.4,1.6,1.7,1.8,1.9,2.0$ & - \\
4 & 3D & 1.0 & 30 & & Figs. 2(a)-2(c) \\
5 & 3D & 1.25 & 30 & & - \\
6 & 3D & 2.0 & 30 & & - \\
7 & 3D & 1.0 & 30 & Random: One layer: $\eta=0.2,0.3,0.35,0.4,0.5,0.6$; two layers: $\eta_{s}=0.2,0.3$ & Figs. 2(d) $-2(\mathrm{f})$ \\
8 & 3D & 1.0 & 30 & Ordered: Dense ordered packing, optimal packing & Figs. 2(g)-2(i) \\
9 & 3D & - & 30 & Frictional flat plane & - \\
10 & 3D & $0.5-2.0$ & $20-28$ & Random: $H_{b} / d=1.0,1.2,1.4,1.6,1.7,1.8,1.9,2.0$ & - \\
11 & 2D & $0.5-2.0$ & 25 & Ordered: $\varepsilon=0.0,0.2,0.4,0.6,0.8,0.9,1.0$ & Figs. 2(j)-2(1) \\
\hline \hline
\end{tabular}

\section{Basal effect on velocity profile}

There are three categories of velocity profile observed in all simulations. In the following, the results of Sets $2,4,6(\Phi=$ $0.5,1.0,2.0$, respectively) are discussed as examples (Fig. 3). The average velocity in the flow direction, $v$, is normalized by the square root of $g d_{p}$, where $g$ is gravitational acceleration. The elevation $y$ is normalized by $d_{p}$. Note that all simulations are performed with the same monodisperse sample [Fig. 1(b)], and only base constructions are different (Fig. 2).

For $\Phi=1.0$ (Set 4) in Fig. 3(a), the base velocity (thus velocity profile) is dependent on the base construction, (a)

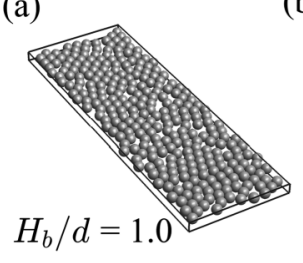

(b)

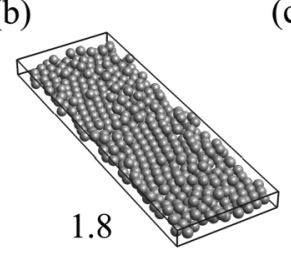

(c)

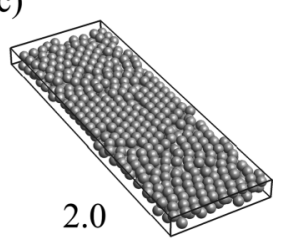

(d)

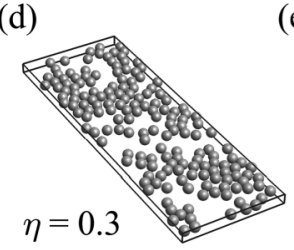

(e)

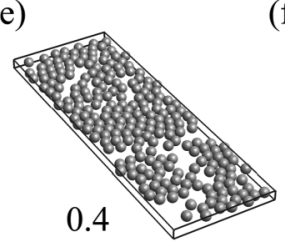

(f)

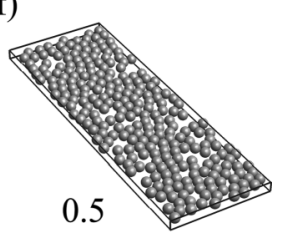

(g)

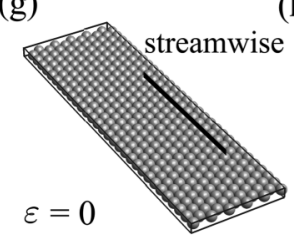

(j)

h)

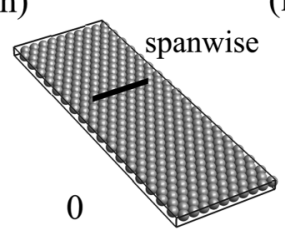

(k)

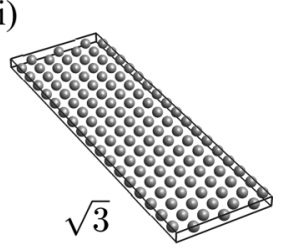

(1)

\section{0}

$\varepsilon=0$

0.4
FIG. 2. Base generation. Random packing in 3D by (a-c) base thickness and (d-f) packing density; regular packing by spacing in ( $\mathrm{g}-$ i) $3 \mathrm{D}$ and $(\mathrm{j}-1) 2 \mathrm{D}$. Note that the regular packing in $(\mathrm{g})$ is streamwise and in (h) is spanwise. Flow is directed down the incline. i.e., the random spacing of the base surface. When the spacing is formed appropriately (e.g., by using $H_{b} / d=$ 1.8 ), sufficient roughness is obtained and no slip occurs at the base. When the spacing is either too large or small, considerable slip is observed. The snapshot taken near the base [Fig. 3(d)] shows that when $H_{b} / d=1.8$, some particles tend to be stopped or decelerated by the bumps. However, the voids are not so deep as to totally capture the flowing particles.

For $\Phi=0.5$ (Set 2) in Fig. 3(b), where the base particles are twice the size of the flowing particles, all cases exhibit an identical nonslip base condition. The result is unaltered by base constructions. Note that the velocity profiles remain zero below the surface of base particles (i.e., the dashed line), which is also seen in [14] when some large and deep voids exist among bumps. By checking the snapshot near the base [Fig. 3(e)], it is clear that in this range particles are trapped in the voids of the base structure. In experimental studies where the flow velocity is measured based on side-view observation, the observable base velocity is nonzero as large base particles enclose the trapped ones. This is referred to as the hole-filling mechanism in [15], where a critical size ratio for the maximum roughness is found in experiments. Further enlarging the base particles beyond the critical size does not promote the roughness, because the voids among large particles are filled by small particles.

For $\Phi=2.0$ (Set 6) in Fig. 3(c), all cases exhibit similar behaviors and the flow is sliding significantly on the relatively small base particles. This observation is essentially unaffected by base constructions. The velocity profile is steeper, implying less interlayer shearing. The snapshot [Fig. 3(f)] shows that the flowing particles can hardly intrude into the base structures.

\section{Necessity to quantify roughness}

In order to focus on the basal effect, we plot the velocity at the bottom of the sample, $v_{b}$, for different base constructions associated with Sets 1-7 in Fig. 4. Base velocity, $v_{b}$, is normalized by surface velocity, $v_{s}$, to eliminate the difference in velocities caused by the different base conditions. The ratio can also be interpreted as the extent of shear propagation from the free surface to the base. Base construction, or its 

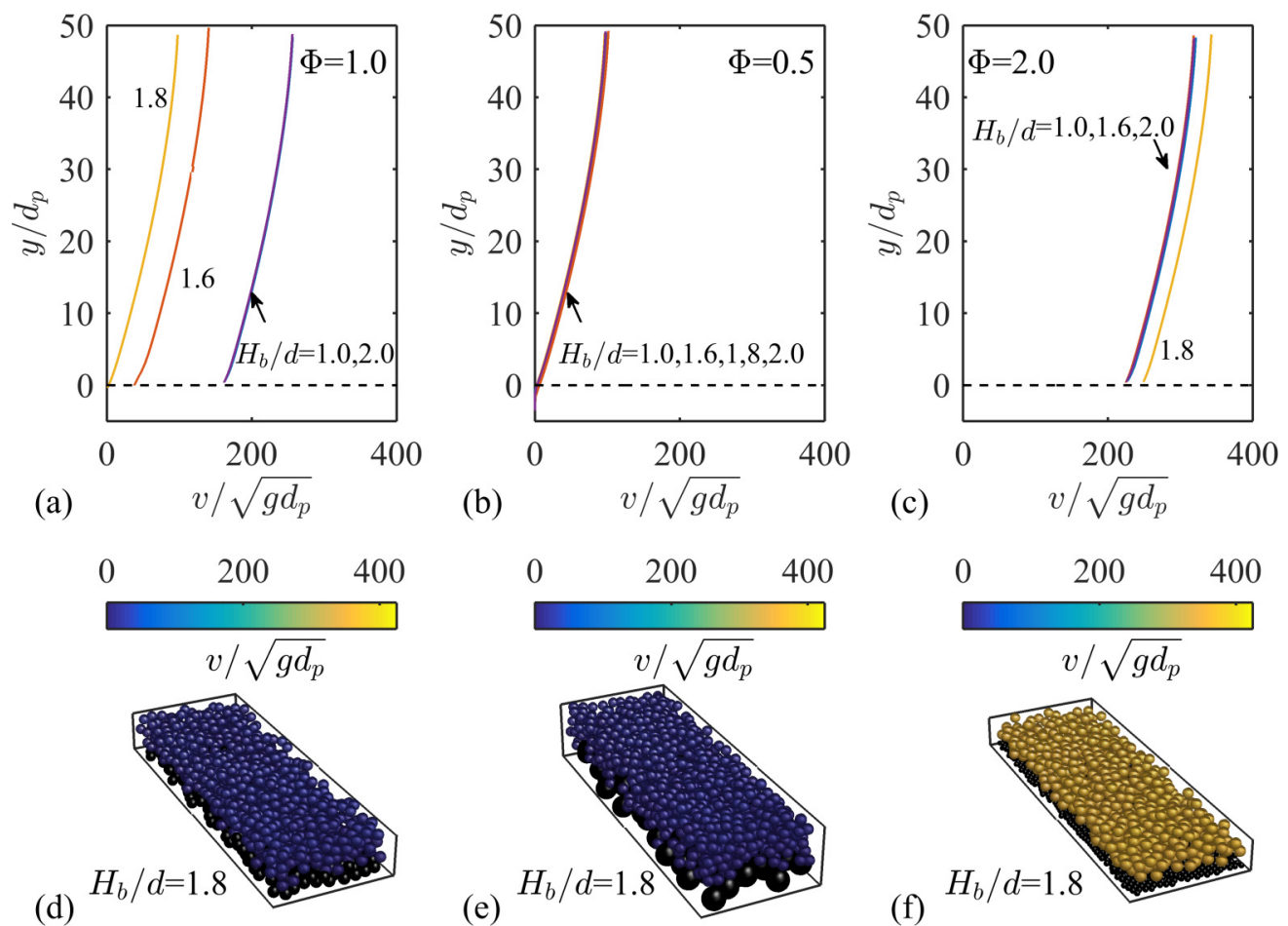

FIG. 3. Basal effect. (a-c) Typical velocity profiles for $\Phi=1.0,0.5,2.0$, respectively. (d-f) Flow snapshots near base (with $H_{b} / d=1.8$ ) for $\Phi=1.0,0.5,2.0$, respectively.

surface morphology, can be indicated by the normalized base thickness, $H_{b} / d$, in Fig. 4(a), or the packing density of base surface, $\eta_{s}$, in Fig. 4(b).

Figure 4 shows that when $\Phi$ is either too large or too small, the base velocity is insensitive to different base constructions. In particular, when $\Phi=2.0$, considerable slip $\left(v_{b} / v_{s} \approx 0.7\right)$ occurs regardless of $H_{b} / d$ and $\eta_{s}$, while $\Phi=0.4,0.5$ always lead to nonslip basal condition. On the other hand, base construction procedures are influential at intermediate size ratios. In Fig. 4(a), when $\Phi=0.67,1.0,1.25, v_{b} / v_{s}$ is generally a function of $H_{b} / d$. A local minimum corresponding to $H_{b} / d=1.8$ can be identified. This indicates that base velocity is the lowest (and nonslip) at a thickness of $1.8 d$ when the base
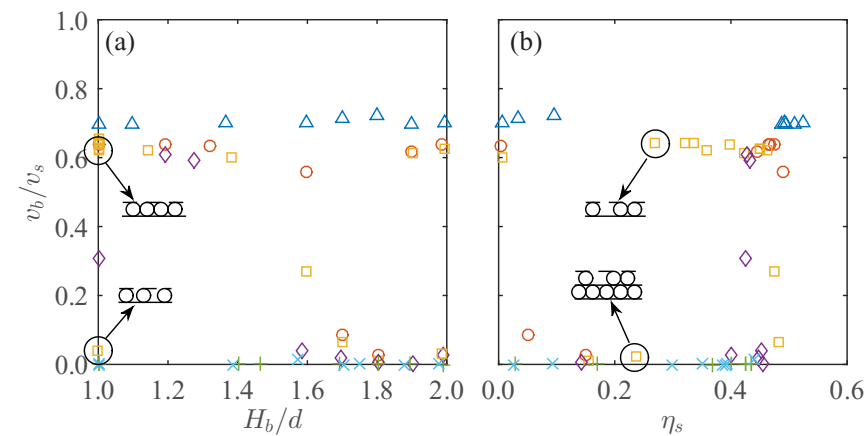

FIG. 4. Base velocity as a function of (a) base thickness and (b) packing density. Symbols represent size ratio: $\Phi=$ 2.0( $\triangle), 1.25(\circ), 1.0(\square), 0.67(\diamond), 0.5(+), 0.4(\times)$. Cases annotated by arrow circles in (a) have the same base thickness but different packing density, and in (b) have similar base surface $\left(\eta_{s}\right)$ but different underneath layers. is generated by the method adopted in Sets 3-5 (Table I). Similarly, a minimum point appears at $\eta_{s} \approx 0.2$ in Fig. 4(b). The nonslip condition associated with the thickness of base layers or the surface density will be elaborated in Sec. III D.

Despite the clear minima around $H_{b} / d=1.8$ and $\eta_{s}=0.2$ for $\Phi=0.67,1.0,1.25$, several issues are revealed by Fig. 4 . Firstly, $v_{b} / v_{s}$ does not depend solely on $H_{b} / d$ or $\eta_{s}$. Size ratio $\Phi$ must be specified to complement the description of basal velocity condition. Similarly, $\Phi$ is also not a sole indicator for base roughness. Secondly, nonunique base velocities exist when $H_{b} / d=1.0$ and 2.0, an example being the annotated cases in Fig. 4(a) for $\Phi=1.0$ (the lower case comes from Set 7). When two bases have the same thickness but one is looser than the other (different $\eta_{s}$ ), their base roughness can be much different [see insets in Fig. 4(a)]. On the other hand, bases with a similar surface density, $\eta_{s}$, can be constructed in many different ways [see insets in Fig. 4(b)]. The two cases selected in Fig. 4(b) have similar packing density at the surface, but one surface is on top of a flat plane while the other is on a dense layer. As the base surface in both cases is loose enough $\left(\eta_{s} \approx 0.28\right)$ to expose its underneath layer to flowing particles, and a flat plane is generally smoother than a dense layer, it is the lower layers that are responsible for the discrepancy of base velocity $\left(v_{b} / v_{s}\right)$ shown in Fig. 4(b). Such a contribution of lower layers has rarely been discussed in previous studies.

To summarize the above discussion, a unique indicator is required to characterize the roughness of a base constructed by spheres, which should (i) simultaneously take into consideration size ratios and base constructions, and (ii) consider bases as an assembly of multiple layers. 
(a)

(b)
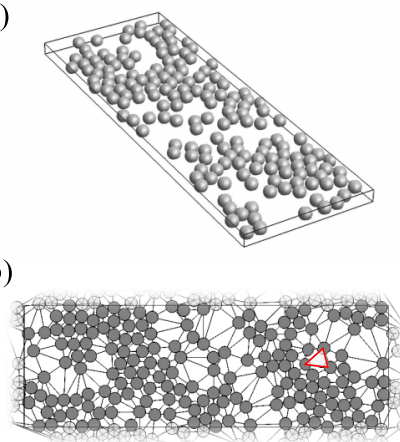

(c)

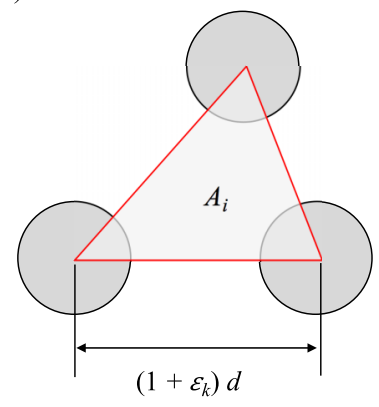

FIG. 5. Discretization of a surface made of spheres. (a) Original layer, (b) projection and discretization, (c) a discretized triangle.

\section{CHARACTERIZATION OF BASE ROUGHNESS}

\section{A. Local roughness}

For clarity, we consider a layer of randomly packed spheres with the same size. The layer is partially filled and the centroids of all particles are coplanar [Fig. 5(a)]. The layer generates a projection on the plane accommodating all centroids, and the circles representing all spheres on the projection plane do not overlap [Fig. 5(b)]. Next we use the Delaunay triangulation (DT) scheme to discretize the plane into triangular patches. The vertices of the triangles are the centers of the circles, and the DT scheme ensures that no centroid is inside any discretized triangles. Note that in Fig. 5(b) ghost particles are placed outside the computational domain due to the use of periodic boundaries. Now the roughness of the layer refers to the statistics of the geometric properties of each individual DT triangle [Fig. 5(c)].

At an arbitrary triangle $i$, the spacing refers to the shortest distances, subtracting the diameter of particles, between any two particles [Fig. 1(a)], and is denoted by $\varepsilon_{k} d$ with $k=1,2,3$ for the three sides in Fig. 5(c). Notice that the area occupied by particles in a triangle is invariant and always equivalent to half a circle's area. The area of the triangle, $A_{i}$, represents the size of the $i$ th local void. Both $\varepsilon_{k} d$ and $A_{i}$ can be adopted to describe the interparticle space.

Now we can define a roughness based on the geometric configuration [Fig. 5(c)] by answering the following question:

If a sphere is placed onto the triangular space $A_{i}$, how stable would it be before it is mobilized by a tangential force in the flow direction?

The concept of stability is linked to the static angle of stability discussed in $[7,8,15]$. In $3 \mathrm{D}$, we consider the flow particle being simultaneously tangential to the three base particles composing the triangular space $A_{i}$ [Fig. 6(a)]. The most stable situation consists of the void positioned in such a way that the centroid of the placed particle is exactly coplanar with the triangle [Fig. 6(b)]. If the void area at this most stable situation is $A_{m}$, the local roughness, $R_{a i}$, is defined by

$$
R_{a i}=\frac{A_{i}}{A_{m}},
$$

where $i$ denotes the $i$ th triangle, and $R_{a}$ stands for the roughness defined by area ratio. It is easy to prove that $A_{m}$ is the area of an equilateral triangle of side length $(1+\Phi) d$. For
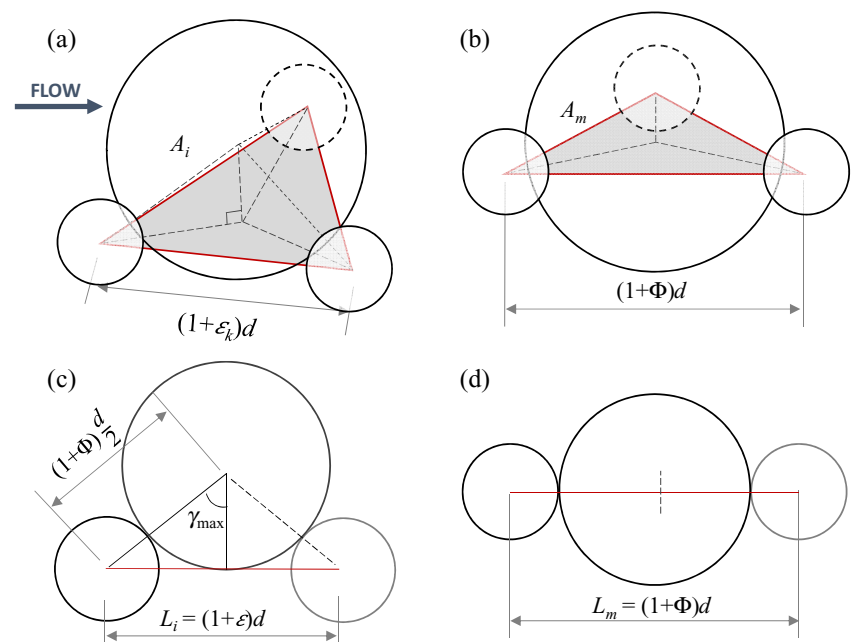

FIG. 6. Definition of local roughness. Larger balls represent flowing particles and smaller balls the fixed base particles. Dashed balls are hidden in 3D. Dashed lines link centroids of balls. The arrow points to the flow direction. (a) An arbitrary void with area $A_{i}$ in $3 \mathrm{D}$, (b) an equilateral void with area $A_{m}$ in 3D (most stable with respect to flow), (c) an arbitrary void with length $L_{i}$ in $2 \mathrm{D}$, (d) a void with length $L_{m}$ in 2D (most stable with respect to flow).

an arbitrary triangle, since the spacing is the most accessible measurement in both numerical and experimental situations, its area can be formulated in terms of the lengths of the three sides, $\left(1+\varepsilon_{k}\right) d$, according to Hero's formula. Thus we have

$$
\begin{aligned}
A_{i} & =d^{2} \sqrt{\frac{\sum_{k}\left(1+\varepsilon_{k}\right)}{2} \prod_{k}\left[\frac{\sum_{k}\left(1+\varepsilon_{k}\right)}{2}-\left(1+\varepsilon_{k}\right)\right]}, \\
A_{m} & =\frac{\sqrt{3}}{4}(1+\Phi)^{2} d^{2} .
\end{aligned}
$$

With Eqs. (2a) and (2b), the definition of local roughness becomes

$$
R_{a i}=\frac{\sqrt{\frac{\sum_{k}\left(1+\varepsilon_{k}\right)}{2} \prod_{k}\left[\frac{\sum_{k}\left(1+\varepsilon_{k}\right)}{2}-\left(1+\varepsilon_{k}\right)\right]}}{\frac{\sqrt{3}}{4}(1+\Phi)^{2}} .
$$

It can be seen that local roughness $\left(R_{a i}\right)$ is a function of spacing $\left(\varepsilon_{k}\right)$ and size ratio $(\Phi)$. Consistent with the observations in Sec. II, a wider spacing or smaller size ratio generally enhances roughness.

In $2 \mathrm{D}$, since the area of a triangle is reduced to the length between two base particles, the local roughness is reduced to

$$
R_{a i}=\frac{L_{i}}{L_{m}}=\frac{1+\varepsilon}{1+\Phi},
$$

where $L_{i}=(1+\varepsilon) d$ is the center-center length of void $i$ [Fig. 6(c)] and $L_{m}=(1+\Phi) d$ is the length at the most stable situation shown in Fig. 6(d). Note that the subscript $k$ is dropped in 2D. Interestingly, Eq. (4) is identical to the maximum contact angle [i.e., $\gamma_{\max }$ in Fig. 6(c)] described in [7], which extends the physical meaning of our definition. Dippel et al. [7] found that a single ball (in 2D) down a rough line can regularly impact with the fixed balls and reach a steady velocity at some inclinations. The average velocity is 


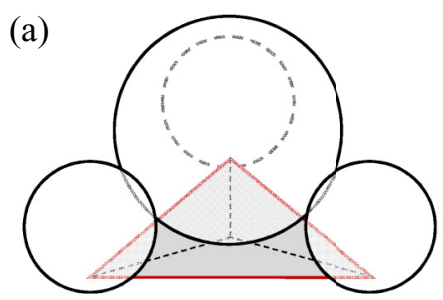

$\Phi \geq 1$

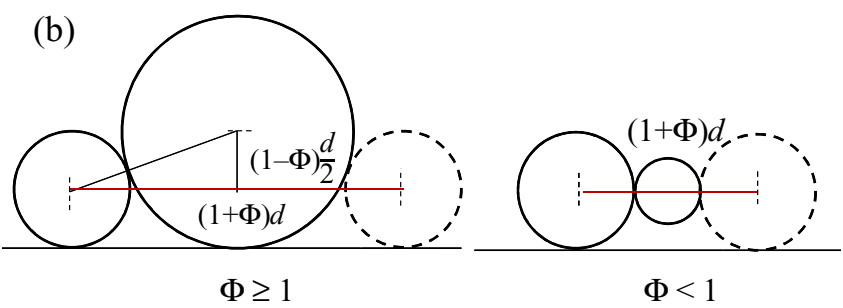

FIG. 7. Critical void area in (a) 3D and (b) 2D situations.

explicitly dependent on $\sin \gamma_{\max }$ [i.e., $R_{a i}$ in Eq. (4)]. When $\sin \gamma_{\max }$ is high, more energy is dissipated at impacts due to normal damping, and the average velocity is lower. In other words, the base is rougher.

\section{B. Multilayer composition}

Next we consider the situation where a base is constructed by multiple layers of particles. In this case, flowing particles can get in contact with lower layers only if the packing density of the surface layer is not too high. This indicates the existence of a critical area $A_{\mathrm{cr}}$ for the local triangular voids, beyond which the contribution of lower layers should be considered. To illustrate the concept of $A_{\mathrm{cr}}$, a reference plane lying beneath the three base particles is used to represent the lower layer [Fig. 7(a)]. The reference plane can refer to either a flat wall or the top edge of the underneath layer. The determination of $A_{\mathrm{cr}}$ is dependent on size ratio. When size ratio $\Phi \geqslant 1$, the critical area $A_{\mathrm{cr}}$ is reached when the flowing particle is simultaneously tangential to all three fixed particles and the reference plane. It can be shown that

$$
A_{\mathrm{cr}}=\frac{3 \sqrt{3}}{4} \Phi d^{2} .
$$

When $\Phi<1$, the critical area $A_{\text {cr }}$ is reached when all centroids are coplanar,

$$
A_{\mathrm{cr}}=A_{m}=\frac{\sqrt{3}}{4}(1+\Phi)^{2} d^{2} .
$$

In $2 \mathrm{D}$, the reference plane is reduced to a line [Fig. 7(b)]. The critical length, $L_{\mathrm{cr}}$, can be written as a function of size ratio $\Phi$. For $\Phi \geqslant 1$,

$$
L_{\mathrm{cr}}=2 \sqrt{\Phi} d
$$

and for $\Phi<1$,

$$
L_{\mathrm{cr}}=L_{m}=(1+\Phi) d .
$$

The critical void size constitutes the basis of multilayer composition. Figure 8 shows a general multilayer situation

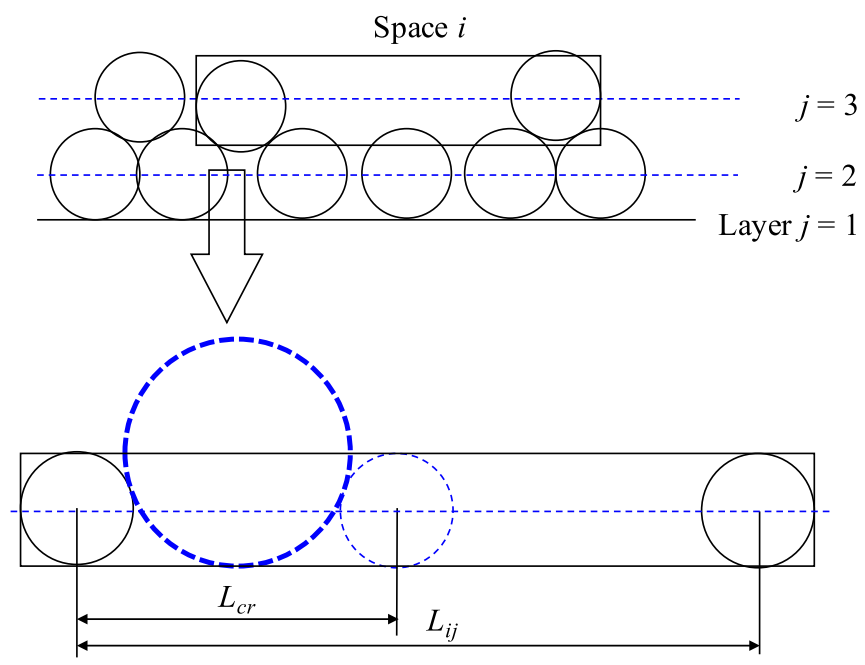

FIG. 8. A base of multiple layers (2D for clarity). Replace $L$ by $A$ in 3D.

where $N_{l}-1$ layer(s) of particles are placed on top of a flat plane. The flat plane is counted as the first layer, as it serves as a substrate of the whole system in both experimental and numerical studies. Typically, $N_{l}=2$ or 3 when the base consists of one or two layer(s) of particles.

Consider a void $i$ in layer $j$, with $A_{i j}$ being its area (Fig. 8). If $A_{i j} \leqslant A_{\mathrm{cr}}$, the portion of layer $j-1$ beneath void $i$ does not contribute to the roughness. As void size increases, i.e., $A_{i j}>$ $A_{\mathrm{cr}}$, a portion of $\left(A_{i j}-A_{\mathrm{cr}}\right) / A_{i j}$ of layer $j-1$ beneath void $i$ is opened to provide roughness. An alternative interpretation is that layer $j-1$ is shielded by void $i$ at a percentage of area $A_{\mathrm{cr}} / A_{i j}$, while the exposed portion of layer $j-1$ is $1-A_{\mathrm{cr}} / A_{i j}$. Therefore, a weight function can be assigned to void $i$ at layer j,

$$
w_{i j}=\min \left(\frac{A_{\mathrm{cr}}}{A_{i j}}, 1\right) .
$$

Here, the min function ensures $w_{i j}<1$ if $A_{i j}>A_{\mathrm{cr}}$ and $w_{i j}=1$ if $A_{i j} \leqslant A_{\mathrm{cr}}$. Correspondingly, the weight function for layer $j-1$ beneath void $i$ is $w_{i, j-1}=1-w_{i j}$. The local roughness, $R_{a i}$, can be extended to the multilayer situation as

$$
R_{a i j}=\min \left(R_{a i}, \frac{A_{\mathrm{cr}}}{A_{m}}\right),
$$

where $R_{a i j}$ is the local roughness of the $i$ th void at the $j$ th layer, $R_{a i}$ is calculated independently from Eq. (3), and the min function indicates that the maximum local roughness, $A_{\text {cr }} / A_{m}$, is reached at the critical area, $A_{\text {cr }}$.

To calculate the roughness of multiple layers, firstly, the arithmetic mean of local roughness, $R_{a i j}$, and local weight function, $w_{i j}$, are obtained over layer $j$,

$$
\begin{aligned}
R_{a j} & =\frac{1}{N_{j}} \sum_{i=1}^{N_{j}} R_{a i j}, \\
w_{j} & =\frac{1}{N_{j}} \sum_{i=1}^{N_{j}} w_{i j},
\end{aligned}
$$


where $R_{a j}$ and $w_{j}$ are the mean roughness and weight function at layer $j$, respectively; $N_{j}$ is the number of triangles at layer $j$; the suffix $i j$ denotes the $i$ th void at the $j$ th layer; and $j=$ $2, \ldots, N_{l}$. When $j=1$ (flat plane), $R_{a j}=0$ and $w_{j}=1$ are immediately imposed. Next, a weighted average is performed to every adjacent two layers in sequence from the bottom two layers $(j=2)$ to the top $\left(j=N_{l}\right)$,

$$
\begin{gathered}
R_{a j} \leftarrow w_{j} R_{a j}+w_{j-1} R_{a, j-1}, \quad j=2, \ldots, N_{l}, \\
R_{a}=R_{a, j=N_{l}},
\end{gathered}
$$

where $R_{a}$ is the roughness of the whole base. Note that Eq. (9) represents an algorithm in a loop manner: It is executed $N_{l}-1$ times as $j$ increases from 2 to $N_{l}$; at each execution, $R_{a j}$ is updated by the result of the current execution; the final value of $R_{a}$ is equal to the result of the last execution. Equation (9) considers the combination of size ratio and base construction [Eqs. (3) and (4)] and the composition of arbitrary multiple layers [Eqs. (7)-(9)]. Note that the formulation of Eq. (9) assumes that the layer-averaged roughness is representative of the entire layer, which is particularly reasonable for a flat plane or a densely packed layer.

The procedure of multilayer composition, i.e., Eqs. (7)(9), remains the same in $2 \mathrm{D}$, except that the void area $A$ is substituted by the spacing length $L$ in Eq. (7).

\section{Range of possible values for $\boldsymbol{R}_{a}$}

The defined roughness, $R_{a}$, is a function of size ratio $(\Phi)$ and spacing $(\varepsilon)$. Figure 9 presents the range of possible values for $R_{a}$. Generally, $R_{a}$ increases as $\Phi$ decreases, while the increase of $\varepsilon$ would not infinitely enhance $R_{a}$ for a given $\Phi$. As $\varepsilon$ increases, an upper bound (UB) for $R_{a}$ is imposed when local voids open up to the critical area $A_{\mathrm{cr}}$. Beyond this point, a nonzero weight is given to the lower, smoother layers. The upper bound is dependent on size ratio $(\Phi)$. When $\Phi \geqslant 1$, the upper bound is $R_{a} \leqslant A_{\mathrm{cr}} / A_{m}$, where $A_{\mathrm{cr}}$ is given by Eq. (5). When $\Phi<1$, the maximum roughness is reached as $A_{\mathrm{cr}}=A_{m}$; hence $R_{a} \leqslant 1$.

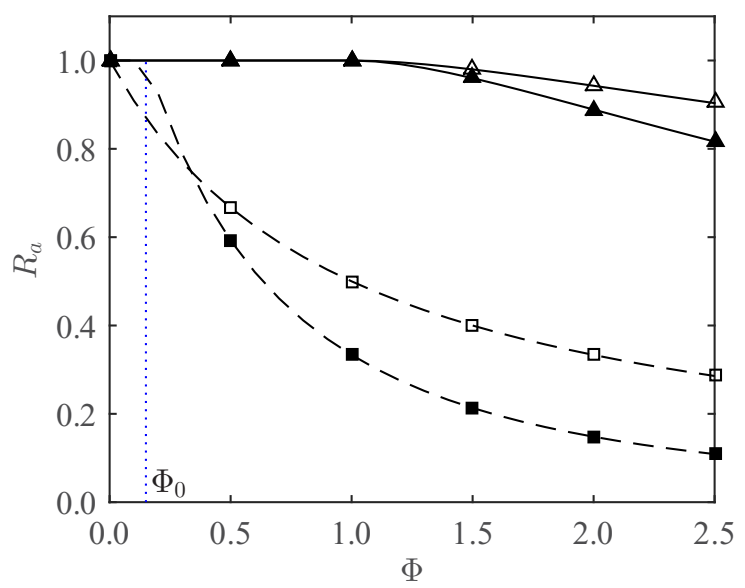

FIG. 9. Values of $R_{a}$ for different size ratio. Dashed lines with filled and empty squares are lower bounds in $3 \mathrm{D}$ and $2 \mathrm{D}$, respectively. Solid lines with filled and empty triangles are upper bounds in 3D and 2D, respectively. Dotted line marks $\Phi_{0}=0.155$.
On the other hand, the lower bound (LB) of $R_{a}$ corresponds to the situation of close packing where no space exists at the center-center lines of base particles; i.e., $\varepsilon=0$ [Figs. 2 (g) and 2(h)]. The lower bound is thus $R_{a i} \geqslant 1 /(1+\Phi)$. However, it is noted that the void cannot be completely closed by spheres in 3D; the minimum void left is equivalent to a sphere of $\Phi_{0}=$ 0.155 . This indicates that when $\Phi \leqslant \Phi_{0}$, we have $R_{a} \equiv 1$; i.e., both upper and lower bounds are 1.0 (Fig. 9).

Figure 9 also illustrates the roughness of a (frictionless) flat plane: As $\Phi$ increases toward infinity, both UB and LB approach zero asymptotically. It indicates that a flat plane is the smoothest in terms of geometric roughness $\left(R_{a}=0\right)$.

The possible range of values for $R_{a}$ is similar in 2D (Fig. 9). For UB, applying Eqs. (4) and (6) yields the following: When $\Phi \geqslant 1, R_{a i} \leqslant 2 \sqrt{\Phi} /(1+\Phi)$; when $\Phi<1, R_{a i} \leqslant 1$. For LB, since the void can be totally closed in 2D [Fig. 1(j)], $R_{a i} \geqslant$ $1 /(1+\Phi)$ is the lower bound of $R_{a}$.

\section{Optimal packing}

As discussed above, the upper bound of $R_{a}$ is reached before the lower layers are exposed to flow particles, since the lower layers (either a flat plane or dense packing) are generally smoother. This indicates the existence of an optimal packing where the maximum roughness is reached for a given size ratio, $\Phi$ (i.e., the UB lines in Fig. 9). Theoretically, the optimal packing is an equally spaced triangular packing [e.g., Fig. 2(i)]. Each triangle from Delaunay triangulation is equilateral with area $A_{\text {cr }}$ [Eq. (5)].

Some equivalent expression of the optimal packing can be derived. For a base with one layer of particles [e.g., Fig. 10(a)], the optimal packing density (corresponding to critical area $A_{\mathrm{cr}}$ ) is

$$
\eta_{\mathrm{cr}}=\frac{\pi}{9 \sqrt{3}} \frac{1}{\Phi},
$$

which is applicable to arbitrary size ratios. When $\Phi=1, \eta_{\mathrm{cr}} \approx$ 0.2 . It confirms the observation in Fig. 4(b) that nonslip basal condition is generally achieved at surface density $\eta_{s} \approx 0.2$, (a)

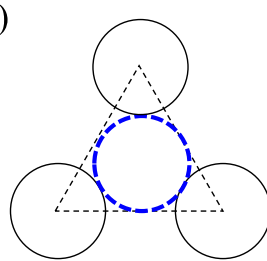

(c)

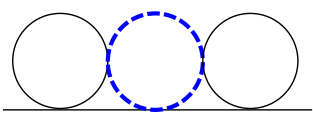

(b)

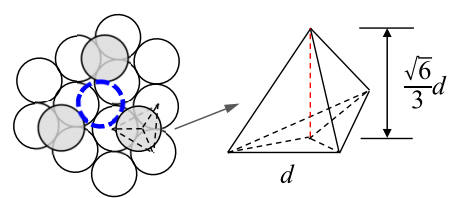

(d)

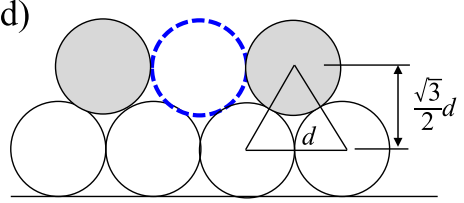

FIG. 10. Typical optimal packing $(\Phi=1)$. Circles with solid and dashed borders are base and flow particles, respectively. Shadows distinguish base particles in different layers. (a) Top view of a onelayer optimal base in 3D. (b) Top view of a two-layer optimal base in $3 \mathrm{D}$, where base thickness is around $1.82 d$. (c) Side view of a one-layer optimal base in 2D. (d) Side view of a two-layer optimal base in 2D, where base thickness is around $1.87 d$. 

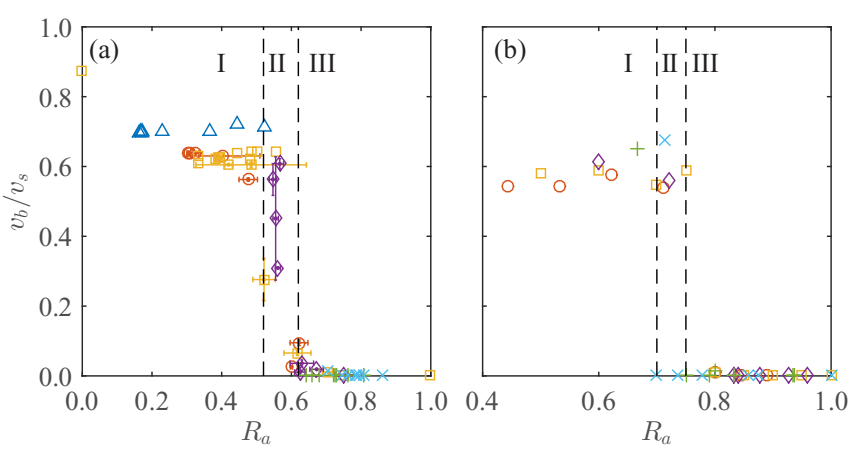

FIG. 11. Transition of slip and nonslip condition in (a) 3D $(\theta=$ $\left.30^{\circ}\right)$ and (b) $2 \mathrm{D}\left(\theta=25^{\circ}\right)$. Symbols represent size ratio $\Phi=2.0(\triangle)$, 1.25( ○), $1.0(\square), 0.67(\diamond), 0.5(+), 0.4(\times)$, respectively. Error bars result from a series of different random seeds in simulations.

provided that base particles are large enough (i.e., $\Phi \leqslant 1.25$ ). For a base with two layers of particles $(\Phi=1)$, if the upper layer follows the optimal packing and the lower layer follows close packing [Fig. 10(b)], it can be shown that the thickness of the base is $H_{b} / d \approx 1.82$. This is associated with the observation in Fig. 4(a), where the lowest base velocity is achieved when $H_{b} / d=1.8$ is adopted in base generations. In $2 \mathrm{D}$, the optimal packing density (by area) is approximately 0.39 for a layer, while the optimal base thickness is $1.87 d$ in a two-layer situation $(\Phi=1)$. Despite the difficulties in constructing the exact optimal packing in experimental and numerical practices, these typical values can still be instructive to the generation of rough bases.

\section{E. Slip and nonslip condition}

Our roughness indicator, $R_{a}$, can predict the occurrence of slip and nonslip condition as a single variable. Figure 11(a) shows the data collected from 3D cases (Sets 1-9), in which three zones can be identified. In Zone I, where $R_{a}=0-0.5$, sliding always occur near the base, where the size ratio can vary from 0.67 to 2.0 . The value $R_{a}=0$ represents the flat plane (Set 9). In Zone III, where $R_{a} \geqslant 0.62$, nonslip condition is always held, where the size ratio can range from 0.4 to 0.67 , as long as the spacing is assigned appropriately. Therefore, a criterion of the imposition of nonslip condition is $R_{a} \geqslant 0.62$. Zone II represents a transition between Zone I and Zone III. A similar $R_{a}$ in this zone (where $\Phi=0.67-1.25$ only) may result in different basal conditions. A series of random seeds are adopted for Sets 3-5 to check repeatability. Error bars indicate more deviations in Zone II, because a looser surface allows more variations in base generation.

A similar phase diagram can be obtained for $2 \mathrm{D}$ cases (Set 11). Note that $\theta=25^{\circ}$ is used for 2D cases, which is close to the limit of steady flow in 2D [28,35]. As shown in Fig. 11(b), the transition is more abrupt in $2 \mathrm{D}$, which means no moderate basal velocity is observed in Zone II in the presented cases. Another observation is that the critical $R_{a}$ beyond which nonslip condition can be held in $2 \mathrm{D}$ (i.e., $R_{a} \geqslant 0.75$ ) is higher than that in $3 \mathrm{D}$ (i.e., $R_{a} \geqslant 0.62$ ). Two reasons are given, the first being that $2 \mathrm{D}$ voids capture particles less efficiently than $3 \mathrm{D}$ voids under the same size ratio, and the second reason is

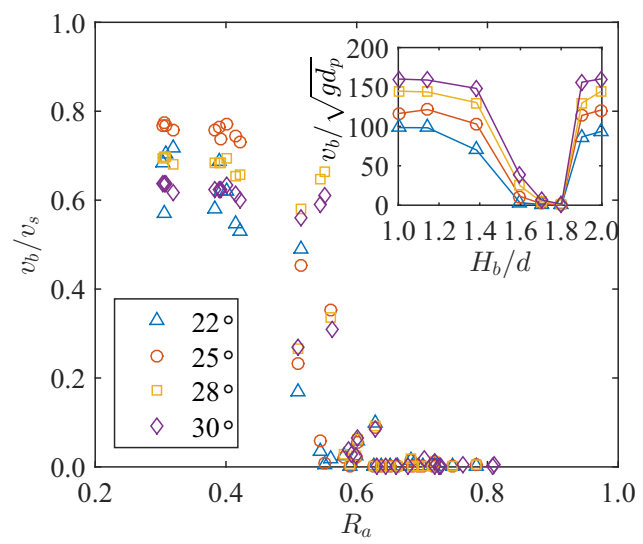

FIG. 12. Slip and nonslip condition at different angles of inclination. Inset: base velocity as a function of base thickness for $\Phi=1.0$ at different inclinations.

the transversal motion of flowing particles near the base in 3D, which causes more energy dissipation [8].

\section{F. Phase diagram}

The angle of inclination is the driving factor for granular chute flows. It defines the kinetic energy gained from potential energy at the steady, fully developed state and the effective friction in the bulk of the flow $[1,2,12,28,29,35,48]$. It is anticipated that the nonslip criterion at a higher inclination should also hold at lower inclinations. A series of different inclinations (i.e., Set 10: $\theta=20^{\circ}, 22^{\circ}, 25^{\circ}, 28^{\circ}$ ) is used to examine the slip and nonslip base condition (Fig. 12). In cases with $\theta=20^{\circ}$ and $R_{a}>0.54$, no flow is observed due to the high roughness and low inclination. Therefore, the collected data at $20^{\circ}$ are excluded from this discussion. For the other values of slope angle, the overall trends of base velocity with increasing $R_{a}$ are all similar to that in Fig. 11(a), which is plotted for $\theta=30^{\circ}$. In the zone where nonslip condition is achieved $\left(R_{a}>0.62\right)$, the influence of $\theta$ is negligible. This proves the robustness of the criterion for nonslip rough base. In the zone where basal velocities are nonzero, the normalized base velocity, $v_{b} / v_{s}$, is randomly influenced by $\theta$. Indeed, the actual magnitude of base velocity, $v_{b}$, is a monotonic function of $\theta$, as shown in the inset of Fig. 12.

The optimal base thickness [Fig. 10(b)] for nonslip condition is also examined for different inclinations. The inset diagram of Fig. 12 presents the basal velocity as a function of base thickness for $\Phi=1.0$ at different angles of inclination. The base velocity, $v_{b}$, attains a minimum value of zero at $H_{b} / d=1.8$, which is independent of the angle of inclination. The optimal thickness, $H_{b} / d=1.8$, is consistent with the theoretical treatment shown in Fig. 10(b). This once again proves that the imposition of nonslip condition is robust as long as an appropriate combination of size ratio and spacing is designated.

Now we further investigate the transition of slip and nonslip condition for different inclinations. An intermediate range of $R_{a}$ is found connecting the regime of sliding $\left(R_{a}<0.51\right)$ and nonslip $\left(R_{a}>0.62\right)$. Two boundaries can be identified to distinguish the three zones similar to Fig. 11(a), which 


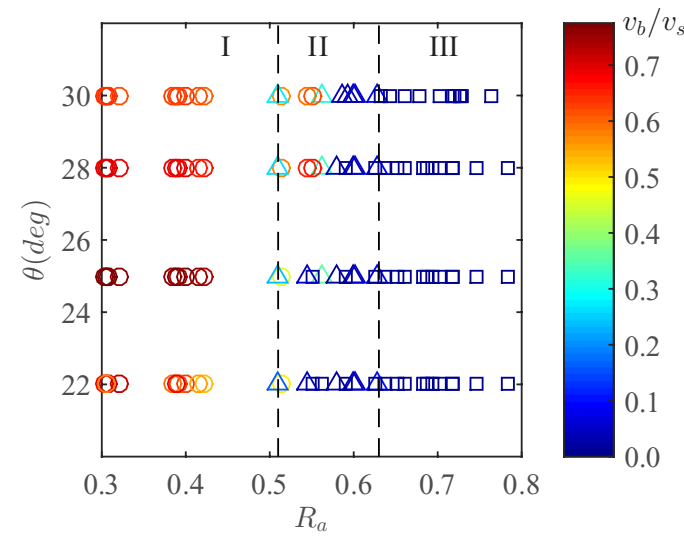

FIG. 13. Phase diagram for different values of slope angle and base roughness. Symbols represent regimes of sliding (circle), transition (triangle), and nonslip (square), respectively. Color map indicates the value of $v_{b} / v_{s}$.

are not influenced by the angle of inclination. A phase diagram can be established taking both inclination and base roughness into consideration (Fig. 13). For all inclinations under consideration, the boundary between the slip and transition regime (Zone I-Zone II) is consistently 0.51 , while the critical roughness defining the nonslip regime (Zone III) is 0.62. Within Zone II, the color map indicates a trend that the nonslip condition can be obtained at smaller values of $R_{a}$ if the angle of inclination is smaller (Fig. 13).

\section{DISCUSSION}

\section{A. Simplified definition}

The definition of roughness is established step by step from a single triangle that composes a small piece of the base. This is mathematically rigorous, but may not be convenient in its implementation. The same concept can be applied in a simpler way, that is, to consider one layer at a time instead of considering the assembly of discretized triangles. The packing density of each layer, which is easier to estimate in practice, can be used to derive a simplified roughness indicator. Let $R_{p}$ be the roughness defined by packing density. The equivalent void area at each layer, $A_{j}$, is derived from packing density, $\eta_{j}$, assuming equilateral triangulation,

$$
A_{j}=\frac{\pi}{12 \eta_{j}} d^{2},
$$

where $j$ is the layer index. The weight function at layer $j$ is determined by $w_{j}=\min \left(A_{\mathrm{cr}} / A_{j}, 1\right)$, while the layer roughness, $R_{p j}$, becomes $R_{p j}=A_{j} / A_{m}$. The multilayer composition remains the same as Eq. (9). The simplified definition may lead to lower resolution in extreme cases where some local areas of the base surface are much denser than other areas. Nevertheless, for a randomly generated base, or a carefully designed ordered base, the simplification is generally acceptable, as examined in Fig. 14.

Figure 14 shows the comparison between the roughness obtained by considering all individual local triangles $\left(R_{a}\right)$ and the roughness obtained by considering only the layers $\left(R_{p}\right)$, for Sets $1-6$. It can be seen that, in general, the data points are lying

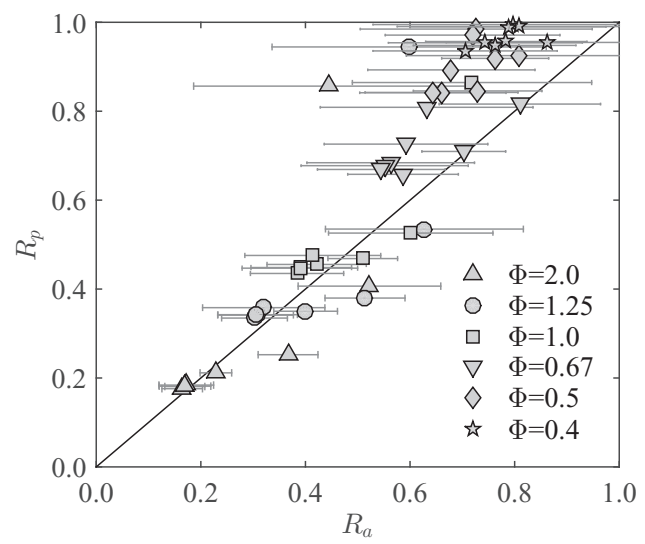

FIG. 14. Simplified definition vs rigorous definition. Horizontal bars represent the range of local roughness, $R_{a i}$, obtained when layer averaging is performed.

near the identity function, which goes through most horizontal bars. The width of horizontal bars indicates the standard deviation of local roughness, $R_{a j}$, obtained when averaging [Eq. (8a)] is performed for layer $j$. It represents the range of deviations rather than errors. In fact, this is the advantage of $R_{a}$ that by considering each individual local triangle, a more rigorous description of the layer can be achieved. Note that in Fig. 14, more data points appear above the 1:1 line, meaning $R_{p}$ tends to overestimate the base roughness. This is attributed to the equilateral assumption made in Eq. (11), as an equilateral triangle has the greatest area in all triangles with the same perimeter. Nonetheless, for the purpose of practical use, evaluation of $R_{p}$ is still recommended as the agreement with $R_{a}$ is generally good. Based on a phase diagram constructed by $R_{p}$ (not shown for brevity) that is similar to Fig. 11, the criterion for nonslip condition is $R_{p}>0.8$.

\section{B. Packing orientation}

The orientation of the packing of base particles may also affect the roughness if a certain packing pattern is followed. For instance, Silbert et al. [14] present two different perfectly ordered bases (denoted as POB1 and POB2) where $\Phi=1.0, \varepsilon=0$. The packing orientation is in streamwise (POB1) or spanwise (POB2) direction. It is found that POB1 is generally smoother than POB2, although both cases have $R_{a}=0.58$ according to Eq. (3). We reproduce the two cases as Figs. 2(g) and 2(h), respectively. For a variety of slope angles $\left(\theta=20^{\circ}-30^{\circ}\right)$, the flows on the streamwise-ordered base are generally faster than those on the spanwise-ordered base (Fig. 15). Although the difference of base velocities is less than $10 \%$, the orientation of local triangles indeed gives rise to a variation of basal condition. This difference caused by packing orientation is omitted in our definition when layer average [Eq. (8)] is performed. As a result, the same $R_{a}$ may refer to different situations distinguished by the flow direction. This indicates that for an ordered surface made of spheres of triangular packing, the surface roughness is anisotropic. Flows in one direction may be easier than in other directions. However, in the current context, the difference 


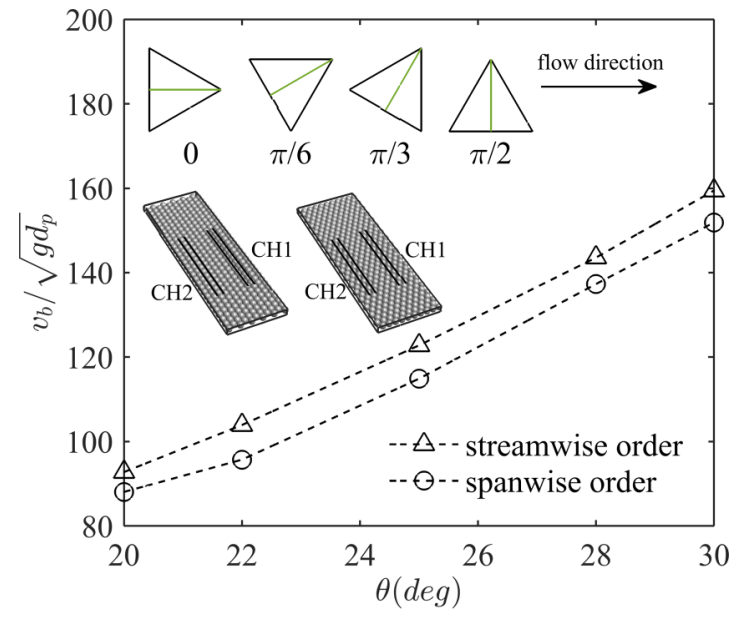

FIG. 15. Effect of packing orientation $\left(\Phi=1.0, \varepsilon=0 ; R_{a}=\right.$ $0.58)$. Streamwise order [also Fig. $2(\mathrm{~g})$ ] is made of triangles at $\pi / 6$ and $\pi / 2$, while spanwise order [also Fig. 2(h)] is made of triangles at 0 and $\pi / 3$, with respect to the flow direction.

is subtle (Fig. 15). For most randomly generated bases in the current study, the packing orientation follows no specific pattern.

To factorize the effect of packing orientation is nontrivial. One way to do so is to consider the channels $(\mathrm{CH} 1$ and $\mathrm{CH} 2$ in Fig. 15) that connect the voids in the flow direction. Let $\beta$ be the packing orientation with respect to the flow direction. When $\beta=0$ and $\pi / 3$, which corresponds to spanwise order, a flowing particle needs to climb up to the top of the fixed particles in order to move from one void to the adjacent void following the channel. The potential of the inherent roughness is fully invoked, thus a factor of 1.0. On the other hand, when $\beta=\pi / 6$ and $\pi / 2$, the minimal elevation a ball needs to escape from one void is characterized by a factor $\cos (\pi / 6)=0.866$. However, the factor of 0.866 is less than the difference found in Fig. $15(\sim 0.95)$. More discussion is needed if packing orientation is the major concern in some context.

\section{CONCLUSION}

In this paper, typical basal conditions encountered in granular chute flows are presented. It is found that the size ratio of flow to base particles and the construction of a bumpy base both determine whether basal slip occurs or not. An alternatively defined indicator of base roughness, $R_{a}$, can quantitatively consider both size ratio and base construction to predict the slip and nonslip condition. It is generalized for random and regular packings of multilayered spheres, in both two- and three-dimensional configurations. The transition of slip and nonslip conditions is well indicated by changes in the value of $R_{a}$. For different inclinations, a phase diagram is established showing the critical value of $R_{a}$, beyond which nonslip condition is respected. Some typical values of $R_{a}$, including its optimization, are provided for practical use. The characterization of base roughness presented in this work can be of great value to future investigations of basal effect in granular flows. For instance, it can be applied to bidisperse flows where the occurrence of size segregation may lead to a wide variety of base roughness.

\section{ACKNOWLEDGMENTS}

The work was supported by Research Grants Council of Hong Kong (under Grant No. RGC/GRF 17203614), the Research Institute for Sustainable Urban Development at The Hong Kong Polytechnic University, and FAP-DF, Brazil. The computation was performed using the HKU Information Technology Services research computing facilities that are supported in part by the Hong Kong UGC Special Equipment Grant (Grant No. SEG HKU09).
[1] GDR MiDi, Eur. Phys. J. E 14, 341 (2004).

[2] R. Delannay, M. Louge, P. Richard, N. Taberlet, and A. Valance, Nat. Mater. 6, 99 (2007).

[3] Y. Forterre and O. Pouliquen, Annu. Rev. Fluid Mech. 40, 1 (2008).

[4] G. H. Ristow, F. X. Riguidel, and D. Bideau, J. Phys. I 4, 1161 (1994).

[5] C. K. K. Lun, Phys. Fluids 8, 2868 (1996).

[6] G. G. Batrouni, S. Dippel, and L. Samson, Phys. Rev. E 53, 6496 (1996).

[7] S. Dippel, G. G. Batrouni, and D. E. Wolf, Phys. Rev. E 54, 6845 (1996).

[8] S. Dippel, G. G. Batrouni, and D. E. Wolf, Phys. Rev. E 56, 3645 (1997).

[9] C. Henrique, M. A. Aguirre, A. Calvo, I. Ippolito, S. Dippel, G. G. Batrouni, and D. Bideau, Phys. Rev. E 57, 4743 (1998).

[10] L. Samson, I. Ippolito, D. Bideau, and G. G. Batrouni, Chaos 9 , 639 (1999).

[11] O. Pouliquen, J. Delour, and S. B. Savage, Nature 386, 816 (1997).
[12] O. Pouliquen, Phys. Fluids 11, 542 (1999).

[13] B. Andreotti and S. Douady, Phys. Rev. E 63, 031305 (2001).

[14] L. E. Silbert, G. S. Grest, S. J. Plimpton, and D. Levine, Phys. Fluids 14, 2637 (2002).

[15] C. Goujon, N. Thomas, and B. Dalloz-Dubrujeaud, Eur. Phys. J. E: Soft Matter Biol. Phys. 11, 147 (2003).

[16] F. da Cruz, S. Emam, M. Prochnow, J.-N. Roux, and F. Chevoir, Phys. Rev. E 72, 21309 (2005).

[17] O. Baran, D. Ertaş, T. C. Halsey, G. S. Grest, and J. B. Lechman, Phys. Rev. E 74, 051302 (2006).

[18] J. J. Drozd and C. Denniston, Phys. Rev. E 78, 041304 (2008).

[19] T. Weinhart, A. R. Thornton, S. Luding, and O. Bokhove, Granular Matter 14, 531 (2012).

[20] A. R. Thornton, T. Weinhart, S. Luding, and O. Bokhove, Eur. Phys. J. E: Soft Matter Biol. Phys. 35, 127 (2012).

[21] V. Kumaran and S. Maheshwari, Phys. Fluids 24, 053302 (2012).

[22] V. Kumaran and S. Bharathraj, Phys. Fluids 25, 070604 (2013).

[23] U. D’ Ortona, N. Thomas, Z. Zaman, and R. M. Lueptow, Phys. Rev. E 92, 062202 (2015). 
[24] U. D'Ortona, N. Thomas, and R. M. Lueptow, Phys. Rev. E 93, 022906 (2016).

[25] D. Berzi and D. Vescovi, Comput. Part. Mech. (2016), doi:10.1007/s40571-016-0115-6.

[26] N. Taberlet, P. Richard, A. Valance, W. Losert, J. Pasini, J. Jenkins, and R. Delannay, Phys. Rev. Lett. 91, 264301 (2003).

[27] R. Artoni and P. Richard, Phys. Rev. Lett. 115, 158001 (2015).

[28] L. E. Silbert, D. Ertaş, G. S. Grest, T. C. Halsey, D. Levine, and S. J. Plimpton, Phys. Rev. E 64, 051302 (2001).

[29] L. E. Silbert, J. W. Landry, and G. S. Grest, Phys. Fluids 15, 1 (2003).

[30] M. Y. Louge, Phys. Rev. E 67, 061303 (2003).

[31] O. Pouliquen, Phys. Rev. Lett. 93, 248001 (2004).

[32] G. Lois, A. Lemaître, and J. M. Carlson, Phys. Rev. E 72, 051303 (2005).

[33] J. M. N. T. Gray and A. R. Thornton, Proc. R. Soc. London, Ser. A 461, 1447 (2005).

[34] P. Jop, Y. Forterre, and O. Pouliquen, Nature 441, 727 (2006).

[35] L. Staron, Phys. Rev. E 77, 051304 (2008).

[36] A. J. Holyoake and J. N. McElwaine, J. Fluid Mech. 710, 35 (2012).

[37] E. Azéma and F. Radjaï, Phys. Rev. Lett. 112, 078001 (2014).
[38] J. L. Baker, T. Barker, and J. M. N. T. Gray, J. Fluid Mech. 787, 367 (2016).

[39] K. van der Vaart, P. Gajjar, G. Epely-Chauvin, N. Andreini, J. M. N. T. Gray, and C. Ancey, Phys. Rev. Lett. 114, 238001 (2015).

[40] L. Jing, F. C. Y. Kwok, and Y. F. Leung, in International Conference on Particle-Based Methods, Fundamentals and Applications, PARTICLES 2015, Barcelona, edited by E. Oñate, M. Bischoff, D. R. J. Owen, P. Wriggers, and T. Zohdi (International Center for Numerical Methods in Engineering (CIMNE), Barcelona, Spain, 2015).

[41] P. Gajjar, K. van der Vaart, A. R. Thornton, C. G. Johnson, C. Ancey, and J. M. N. T. Gray, J. Fluid Mech. 794, 460 (2016).

[42] S. B. Savage and K. Hutter, J. Fluid Mech. 199, 177 (1989).

[43] R. M. Iverson, Rev. Geophys. 35, 245 (1997).

[44] C. G. Johnson, B. P. Kokelaar, R. M. Iverson, M. Logan, R. G. LaHusen, and J. M. N. T. Gray, J. Geophys. Res. (2012), doi:10.1029/2011JF002185.

[45] A. Tripathi and D. V. Khakhar, Phys. Fluids 23, 113302 (2011).

[46] L. Staron and J. C. Phillips, Phys. Rev. E 92, 022210 (2015).

[47] L. Staron and J. C. Phillips, Phys. Fluids 26, 33302 (2014).

[48] O. Pouliquen and F. Chevoir, C. R. Phys. 3, 163 (2002). 